\title{
ON THE COMPLETION OF HAUSDORFF LOCALLY SOLID RIESZ SPACES(1)
}

BY

\section{CHARALAMBOS D. ALIPRANTIS}

ABSTRACT. In this paper we consider Hausdorff locally solid Riesz spaces $(L, \tau)$ and we denote by $(\hat{L}, \hat{\tau})$ the Hausdorff topological completion of $(L, \tau)$. It is proved that $(\hat{L}, \hat{\tau})$ is a Hausdorff locally solid Riesz space containing $L$ as a Riesz subspace. We study the properties of $(L, \tau)$ which are inherited by $(\hat{L}, \hat{\gamma})$.

0. Introduction. It is known that every Hausdorff topological vector space $(E, r)$ has a unique, up to a topological and algebraic isomorphism, Hausdorff topological completion $(\hat{E}, \hat{\tau})$, i.e., there exists a complete Hausdorff topological vector space $(\hat{E}, \hat{\gamma})$ such that $(E, r)$ is a $\hat{\tau}$-dense subspace of $(\hat{E}, \hat{\gamma})$ (see $[12$, p. 17] or [4, p. 131]). It is also known that if $\{V\}$ is a neighborhood basis of the r-neighborhoods of zero then $\{\bar{V}\}$, where $\bar{V}$ is the $\hat{r}$-closure of $V$ in $\hat{E}$, is also a neighborhood basis for the $\hat{\gamma}$-neighborhoods of zero in $(\hat{E}, \hat{\tau})$ (see $[12$, p. 17]), which in particular implies that $(\hat{E}, \hat{\gamma})$ is metrizable if, and only if, $(E, r)$ is metrizable.

In the following we shall assume that $(L, r)$ is a Hausdorff locally solid Riesz space with its Hausdorff topological completion denoted by $(\hat{L}, \hat{\tau})$. In this paper we shall investigate the properties of $(L, \gamma)$ which are inherited by $(\hat{L}, \hat{\tau})$.

This problem, in the case of the normed Riesz spaces, has been investigated by W. A. J. Luxemburg [7]. In the case of Hausdorff locally convex, locally solid Riesz spaces some results have been obtained by M. Duhoux [2] and I. Kawai [5]. Some other interesting results have been obtained by D. H. Fremlin in [3].

1. Notation and basic concepts. For notation and terminology concerning Riesz spaces we follow [9]. Let $L$ be a Riesz space. A vector subspace $A$ is called a Riesz subspace if for every $u, v \in A$ the element $u \bigvee v$ (taken in $L$ ) is in $A$. A vector subspace $A$ is called an ideal if $|u| \leq|v|$ and $v \in A$ implies $u \in A$. An ideal $A$ is called a $\sigma$-ideal if $\theta \leq u_{n} \uparrow u$ in $L$ and $\left\{u_{n}\right\} \subseteq A$ implies $u \in A$. An

Presented to the Society, January 15, 1974; received by the editors May 25, 1973. AMS (MOS) subject classifications (1970). Primary 46A40; Secondary 46A15.

Key words and phrases. Topological completion, locally solid Riesz spaces, order and topological continuity.

(1) This paper contains results from the author's Ph. D. dissertation at the California Institute of Technology (April 1973). The author wishes to thank his advisor, Professor w. A. J. Luxemburg, for his help. 
ideal $B$ is called a band if $\theta \leq u_{a} \uparrow u$ in $L$ and $\left\{u_{a}\right\} \subseteq B$ implies $u \in B$. A band $B$ is called a principal band if there exists $u \in B$ such that $B$ is the smallest band containing $u$, i.e., if it follows from $u \in A$ and $A$ is a band that $B \subseteq A$. In this case we denote $B$ by $B_{u}$.

A Riesz space $L$ is called $\sigma$-Dedekind complete if every nonempty countable subset bounded from above in $L$ has a supremum, or equivalently, if $\theta \leq u_{n} \uparrow \leq u_{0}$ in $L$ implies $u_{n} \uparrow u$ for some $u$ in $L . L$ is called Dedekind complete if every nonempty subset of $L$ bounded from above has a supremum, or equivalently, if $\theta \leq$ $u_{a} \uparrow \leq u_{0}$ in $L$ implies $u_{a} \uparrow u$ for some $u$ in $L$. Finally $L$ is called super Dedekind complete if it is Dedekind complete and if $\theta \leq u_{a} \uparrow u$ in $L$ implies $u_{a_{n}} \uparrow u$ for some sequence $\left\{u_{a_{n}}\right\} \subseteq\left\{u_{a}\right\}$.

A Riesz space $L$ is called Archimedean if $\theta \leq n u \leq v$ for $n=1,2, \ldots$ and some $u, v \in L^{+}$implies $u=\theta$.

$A$ band $B$ of a Riesz space $L$ is called a projection band if $B \oplus B^{d}=L$, where $B^{d}=\{u \in L:|u| \wedge|\nu|=\theta$ for all $v \in B\}$. The Riesz space $L$ is said to have the projection property if every band is a projection band. The Riesz space $L$ is said to have sufficiently many projections if every nonzero band contains a nonzero projection band. Finally, $L$ is said to have the principal projection property if every principal band of $L$ is a projection band.

A subset $S$ of $L$ is called a solid set if $|u| \leq|v|$ and $v \in S$ implies $u \in S$. If $r$ is a linear topology of $L$ (a topology for which both mappings $(u, v) \mapsto u+v$, $(\lambda, u) \mapsto \lambda u$ are continuous) with a basis for the neighborhood system of the origin consisting of solid sets, then $(L, r)$ is called a locally solid linear topological Riesz space, or briefly, a locally solid Riesz space.

Following W. A. J. Luxemburg and A. C. Zaanen [8, Note X] we use the following conditions for locally solid Riesz spaces $(L, \tau)$ :

$(\mathrm{A}, 0): u_{n} \downarrow \theta$ and $\left\{u_{n}\right\}$ r-Cauchy implies $u_{n} \stackrel{r}{\rightarrow} \theta$,

$(\mathrm{A}, \mathrm{i}): u_{n} \downarrow \theta$ implies $u_{n} \stackrel{\tau}{\rightarrow} \theta$,

(A, ii): $u_{a} \downarrow \theta$ implies $u_{a} \stackrel{\tau}{\rightarrow} \theta$,

( $A$, iii): $\theta \leq u_{n} \uparrow \leq u$ implies that $\left\{u_{n}\right\}$ is a $r$-Cauchy sequence, i.e., every order bounded increasing sequence in $L$ is a $r$-Cauchy sequence,

(A, iv): $\theta \leq u_{a} \uparrow \leq u$ implies that $\left\{u_{a}\right\}$ is a $\tau$-Cauchy net, i.e., every order bounded increasing net is a $r$-Cauchy net,

(B, i): $\theta \leq u_{n} \uparrow$ and $\left\{u_{n}\right\} r$-bounded implies that $\left\{u_{n}\right\}$ is a $r$-Cauchy net,

(B, ii): $\theta \leq u_{\alpha} \uparrow$ and $\left\{u_{\alpha}\right\}$ r-bounded implies that $\left\{u_{\alpha}\right\}$ is a $r$-Cauchy net.

(A subset $S$ of a topological vector space $(E, \tau)$ is called $r$-bounded if for every neighborhood $V$ of the origin there exists a positive number $\lambda>0$ (depending on $V$ ) such that $\lambda A \subseteq V$ (see $[4, \mathrm{p} .108])$.)

The next theorem gives some information about the interrelation of the above properties. 
Theorem 1.1. For locally solid Riesz spaces the following bold:

(i) $(\mathrm{A}, \mathrm{ii}) \Rightarrow(\mathrm{A}, \mathrm{i}) \Rightarrow(\mathrm{A}, 0)$.

(ii) $(L, \tau)$ satisfies (A, iii) if, and only if, $(L, r)$ satisfies $(A, i v)$.

(iii) $(L, r)$ satisfies $(\mathrm{B}, \mathrm{i})$ if, and only if, $(L, \tau)$ satisfies $(\mathrm{B}, \mathrm{ii})$.

(iv) $(B, i) \Rightarrow(A$, iii).

(v) If $L$ is Archimedean, then $(A, \mathrm{ii}) \Rightarrow(\mathrm{A}$, iii).

See [1, Theorem 2.1].

Next the above properties are illustrated with examples.

Example 1.2. (i) Consider the Riesz subspace $L$ of $l_{\infty}$ consisting of all sequences which are eventually constant, i.e., $f=\left\{x_{n}\right\}$ is in $L$ if there exists a constant $c$ and an integer $n_{0}$ (both depending on $f$ ) such that $x_{n}=c$ for all $n \geq n_{0}$. Let $r$ be the Hausdorff locally solid topology generated on $L$ by the sup norm. Then $r$ satisfies $(A, 0)$, but $(A, i),(A, i i i)$ and $(B, i)$ do not hold. Note that $r$ is not complete.

(ii) Consider the Riesz space $L$ of all real sequences which are eventually zero, i.e., $f=\left\{x_{n}\right\}$ is in $L$ if there is $n_{0}$ (depending on $f$ ) such that $x_{n}=0$ for all $n \geq n_{0}$, with the pointwise ordering. Let $r$ be the Hausdorff locally solid topology generated by the sup norm. Then $(L, r)$ satisfies $(A, i i)$ and $(A$, iii) but does not satisfy $(B, i)$. Note that $r$ is not complete.

(iii) Let $L=C_{[0,1]}$, i.e., let $L$ be the Riesz space of all real valued continuous functions defined on $[0,1]$ with the pointwise ordering and let $r$ be the Hausdorff locally solid topology generated by the norm $\rho(u)=\int_{0}^{1}|u(x)| d x$. Then $(B, i)$ and $(A$, iii) hold, but $(A, 0)$ does not hold [9, Exercise $18.14(i)$, p. 104]). Note that $r$ is not complete.

(iv) Let $L$ be the Riesz space of all real valued functions defined on an uncountable set $X$ and such that for every $f \in L$ there exists a real number $f(\infty)$ such that, given any $\epsilon>0$, we have $|f(x)-f(\infty)|>\epsilon$ for finitely many $x$. In other words, $L=C\left(X_{\infty}\right)$, where $X_{\infty}$ is the one-point compactification of the set $X$, topologized with the discrete topology. Let $r$ be the Hausdorff locally solid topology generated by the norm $\rho(u)=\sup \{|u(x)|: x \in X\}$. Then $(L, r)$ satisfies $(A, i)$, but ( $A, \mathrm{ii}),(A, \mathrm{iii})$ and $(B, i)$ do not hold. Note that $r$ is complete.

(v) Let $0<p<1$ and let $L$ be the real vector space of all real valued Lebesgue measurable functions $f$ defined on $[0,1]$ such that $\int_{0}^{1}|f(t)|^{p} d t<+\infty$. Then $L$ becomes a Riesz space under the ordering $f \leq g$, whenever $f(x) \leq g(x)$ for all $x \in[0,1]$. Now, given $n \in N, \delta>0$ and $F=\left\{x_{1}, \cdots, x_{k}\right\} \subseteq[0,1]$, define the set:

$$
W_{F, n, \delta}=\left\{u \in L: \int_{0}^{1}|u(x)|^{p} d x<1 / n \text { and }\left|u\left(x_{i}\right)\right|<\delta, \text { for } i=1, \cdots, k\right\} .
$$

For $F$ running over the finite subsets of $[0,1], n$ over $N$ and $\delta$ over $(0,+\infty)$ we 
get a family of sets $\left\{W_{F, n, \delta}\right\}$ which is a filter basis for a neighborhood system of the origin for a uniquely determined linear topology $r$ of $L[4$, p. 81]. Obviously each $W_{F, n, \delta}$ is a solid set. It is also evident that $r$ is a Hausdorff topology, so $(L, r)$ is a Hausdorff locally solid Riesz space.

Then we can verify the following properties:

(1) $r$ is a sequentially complete but not a complete topology.

(2) $(A, i),(B, i)$ and $(A$, iii) hold, but $(A$, ii) does not hold. (See [1].)

(vi) Let $L=R^{X}$, where $X$ is a nonempty set, and let $\tau$ be the product topology. Then $(L, r)$ is a Hausdorff locally solid Riesz space satisfying (A, ii), $(A, \mathrm{iii})$ and $(B, i)$ conditions. Here $r$ is complete.

(vii) The product of the spaces in examples (i) and (ii) with the product topology gives a Hausdorff locally solid Riesz space $(L, r)$, in which $(A, 0),(A, i i i)$ and $(B, i)$ do not hold. Note that $r$ is not complete. $\square$

Note. It is easy to see that $(A, \mathrm{iii})$ is equivalent to the statement: "Every sequence $\left\{u_{n}\right\} \subseteq L$ with $\theta \leq u_{n} \downarrow$ is a $r$-Cauchy sequence."

The following two lemmas will be useful later.

Lemma 1.3. Let $(L, \gamma)$ be a Hausdorff locally solid Riesz space. Then we bave:

(i) $L$ is an Arcbimedean Riesz space.

(ii) If $u_{a} \uparrow$ and $u_{a} \stackrel{\tau}{\rightarrow} u$, then $u_{a} \uparrow u$ in L. A similar statement bolds for decreasing nets.

(iii) Every band of $L$ is r-closed.

Proof. First we note that since $r$ is a Hausdorff topology $L^{+}$is r-closed, simply because $L^{+}=\left\{u \in L: u^{-}=\theta\right\}$.

(i) Let $\theta \leq n u \leq v$ for $n=1,2, \ldots$ and some $u, v \in L^{+}$.

Then we have $\theta \leq v / n-u \stackrel{r}{\rightarrow}-u \in L^{+}$, which implies $u=\theta$.

(ii) For fixed $a_{0}$ we have $u_{a}-u_{a_{0}} \geq \theta$ for all $\alpha \geq a_{0}$.

It follows from this that $u-u_{a_{0}} \geq \theta$. This shows that $u$ is an upper bound of $\left\{u_{a}\right\}$. Now if $u_{a} \leq v$ for all $a$, then $\theta \leq v-u_{a} \stackrel{r}{\rightarrow} v-u$. Hence $u \leq v$. This shows that $u_{a} \uparrow u$.

(iii) Let $D$ be a nonempty subset of $L$ and let $u \in \overline{D^{d}}$, where $D^{d}=\{v \in L$ : $|v| \wedge|w|=\theta$ for all $w \in D\}$. Then there exists a net $\left\{u_{a}\right\} \subseteq D^{d}$ such that $u_{a} \stackrel{r}{\rightarrow} u$. Now if $w \in D$ it follows that $\theta=\left|u_{a}\right| \wedge|w| \stackrel{r}{\rightarrow}|u| \wedge|w|$ and hence (since $r$ is a Hausdorff topology) $|u| \wedge|w|=\theta$, i.e., $u \in D^{d}$. This shows that $D^{d}$ is r-closed. Since every band $A$ of $L$ satisfies $A=A^{d d}$ [9, Theorem 22.3, p. 114] it follows easily that every band of $L$ is r-closed.

Lemma 1.4. Let $(L, r)$ be a Hausdorff locally solid Riesz space. Assume tbat for the two nets $\left\{f_{\alpha}\right\},\left\{g_{a}\right\}$ of $L$ we bave $\theta \leq f_{a} \downarrow \leq g_{\alpha} \downarrow$ and $g_{a}-f_{a} \stackrel{r}{\rightarrow} \theta$. 
Then $f_{a} \downarrow f$ in $L$ if, and only if, $g_{a} \downarrow f$ in $L$.

Proof. Assume first $f_{a} \downarrow f$ in $L$. Let $g_{a} \geq g \geq f$ for all $a$. Then $g_{a}-f_{a}=$ $\left(g_{a}-f_{a}\right)^{+} \geq\left(g-f_{a}\right)^{+} \geq \theta$. It follows from this that $\left(g-f_{a}\right)^{+} \stackrel{r}{\rightarrow} \theta$. But, $\theta \leq\left(g-f_{a}\right)^{+} \uparrow$ $(g-f)^{+}=g-f$. Hence from Lemma 1.3 (ii) we get $g-f=\theta$, i.e., $g_{a} \downarrow f$. Now, if $g_{a} \downarrow f$ in $L$, then we have $\theta \leq f-f \wedge f_{a} \uparrow \leq g_{a}-f_{a} \stackrel{r}{\rightarrow} \theta$. So $f-f \wedge f_{a} \stackrel{r}{\rightarrow} \theta$. It follows from Lemma 1.3(ii) that $f-f \wedge f_{a}=\theta$ for all $a$, so $f \leq f_{a}$ for all $a$. Since $g_{a} \downarrow f$ and $f_{a} \leq g_{a}$ for all $\alpha$ it follows easily that $f_{a} \downarrow f_{\text {. }}$

2. The completion space $(\hat{L}, \hat{\gamma})$. We recall that by $(L, \tau)$ we shall denote a Hausdorff locally solid Riesz space and by $(\hat{L}, \hat{r})$ its Hausdorff topological comple: tion. We shall call $(\hat{L}, \hat{r})$ the topological completion of $(L, r)$.

We start with the following important theorem.

Theorem 2.1. Let $(L, r)$ be a Hausdorff locally solid Riesz space and let $(\hat{L}, \hat{\tau})$ be the topological completion of $(L, \tau)$. If $\hat{L}^{+}$denotes the $\hat{r}$-closure of $L^{+}$ in $\hat{L}$, then $\hat{L}^{+}$is a cone of $\hat{L}$, and $(\hat{L}, \hat{r})$ equipped with this cone is a Hausdorff locally solid Riesz space. In particular it follows that $L$ is a Riesz subspace of $\hat{L}$.

Proof. We show first that $\hat{L}^{+}$(= the $\hat{\tau}$-closure of $L^{+}$in $\hat{L}$ ) is a cone of $\hat{L}$. Since $L^{+}+L^{+} \subseteq L^{+}$and $a L^{+} \subseteq L^{+}$for all $a \geq 0$, it follows easily from the continuity of the addition and the multiplication that

$$
\hat{L}^{+}+\hat{L}^{+} \subseteq \hat{L}^{+} \text {and } a \hat{L}^{+} \subseteq \hat{L}^{+} \text {for all } a \geq 0 \text {. }
$$

So, we have only to show that $\hat{L}^{+} \cap-\hat{L}^{+}=\{\theta\}$. To this end, let $\hat{u} \in \hat{L}^{+} \cap-\hat{L}^{+}$. Then there are two nets $\left\{u_{a}\right\},\left\{w_{\beta}\right\} \subseteq \cdot L^{+}$such that $u_{a} \stackrel{\hat{q}}{\rightarrow} \hat{u}, w_{\beta} \stackrel{\vartheta}{\rightarrow}-\hat{u}$. Thus

$$
\theta \leq u_{\alpha} \leq u_{\alpha}+w_{\beta} \frac{r}{(\alpha, \beta)} \theta \text {. }
$$

Since $r$ is a locally solid topology we see that $u_{a} \stackrel{\hat{r}}{\rightarrow} \theta$. Hence $\hat{u}=\theta$, since $\hat{r}$ is also a Hausdorff topology This completes the proof that $\hat{L}^{+}$is a cone of $\hat{L}$.

We show next that $\left(\hat{L}, \hat{L}^{+}\right)$is a Riesz space. The mapping $u \mapsto u^{+}$from $(L, r)$ into $(\hat{L}, \hat{r})$ is a uniformly continuous mapping. Hence, since $(\hat{L}, \hat{r})$ is complete, it can be extended uniquely to a uniformly continuous mapping $p$ from $\hat{L}$ into $\hat{L}$. We show next that $p(\hat{f})=\hat{f} \vee \theta$ in $\hat{L}$. To this end, let $\hat{f} \in \hat{L}$. Then $f_{\alpha} \stackrel{\hat{f}}{\rightarrow} \hat{f}$ for some net $\left\{f_{a}\right\}$ of $L$. It follows from $f_{a}^{+} \geq f_{a}$ that $p\left(f_{a}\right)-f_{a}=f_{a}^{+}-f_{a} \in L^{+}$, which implies $p(\hat{f})-\hat{f} \in \overline{L^{+}}=\hat{L}^{+}$, i.e., $p(\hat{f}) \geq \hat{f}$ in $\hat{L}$. Obviously, we also have that $p(\hat{f})$ $\epsilon \hat{L}^{+}$. Now let $\hat{f} \leq \hat{g}$ and $\theta \leq \hat{g}$ in $\hat{L}$. We pick two nets $\left\{f_{a}\right\},\left\{g_{a}\right\}$ of $L^{+}$such that $f_{a} \stackrel{\hat{\tau}}{\rightarrow} \hat{g}-\hat{f}$ and $g_{a} \stackrel{\hat{\tau}}{\rightarrow} \hat{g}$, then we have $b_{a}=g_{a}-f_{a} \stackrel{\hat{\tau}}{\rightarrow} \hat{f}$. But then $g_{a}=g_{a}^{+} \geq b_{a}^{+}$, so $g_{a}-p\left(b_{a}\right) \in L^{+}$and from this we get (by taking the $\hat{r}$-limits) that $\hat{g}-p(\hat{f}) \in \hat{L}^{+}$, i.e., $\hat{g} \geq p(\hat{f})$ in $\hat{L}$. This shows that $p(\hat{f})=\hat{f}^{+}$in $\hat{L}$, and this shows that $\left(\hat{L}, \hat{L}^{+}\right)$ is a Riesz space.

We finally show that $(\hat{L}, \hat{\tau})$ is a locally solid Riesz space. To this end let 
$\bar{V} \in\{\bar{V}\}$. Pick $\bar{W} \in\{\bar{V}\}$ such that $\bar{W}+\bar{W} \subset \bar{V}$. Choose next $\bar{W}_{1} \in\{\bar{V}\}$ such that $\bar{W}_{1}+$ $\bar{W}_{1} \subseteq \bar{W}$. Since $u \mapsto u^{+}$is continuous there exists $\bar{U} \in\{\bar{V}\}$ such that $\hat{u} \in \bar{U}$ implies $\hat{u}^{+} \in \bar{W}_{1}$. Since we can suppose that our original basis $\{V\}$ of $(L, \tau)$ consists of solid sets, $\{\bar{V}\}$ will be consisting of circled sets. But then $\hat{v} \in \bar{U}$ implies $-\hat{v} \in \bar{U}$ and so $(-\hat{v})^{+}=\hat{v}^{-} \in \bar{W}_{1}$. Thus

$$
|\hat{v}|=\hat{v}^{+}+\hat{v}^{-} \epsilon \bar{W}_{1}+\bar{W}_{1} \subseteq \bar{W}
$$

Hence $\hat{v} \in \bar{U}$ implies $|\hat{v}| \epsilon \bar{W}$. Now let $|\hat{w}| \leq|\hat{v}|$ with $\hat{v} \in \bar{U}$. Then obviously we have that $\hat{w}^{+}$and $\hat{w}^{-}$are in $\bar{W}$ and hence $\hat{w}=\hat{w}^{+}-\hat{w}^{-} \epsilon \bar{W}+\bar{W} \subseteq \bar{V}$. This shows that the solid hull $S(\bar{U})$ of $\bar{U}$ is contained in $\bar{V}$, i.e., $\bar{U} \subseteq S(\bar{U}) \subseteq \bar{V}$. This shows that $(\hat{L}, \hat{\tau})$ is a locally solid Riesz space.

The next theorem gives a characterization for $L$ to be an ideal of $\hat{L}$.

Theorem 2.2. Let $(\hat{L}, \hat{\gamma})$ be the topological completion of the Hausdorff locally solid Riesz space $(L, \tau)$. Then the following statements are equivalent.

(i) $L$ is an ideal of $\hat{L}$.

(ii) Every order interval of $L$ is $r$-complete.

Proof. (i) $\Rightarrow$ (ii). Since $[f, g]=f+[\theta, g-f]$ it is enough to show that every interval $[\theta, u]\left(u \in L^{+}\right)$is $r$-complete. So let $\left\{f_{a}\right\}$ be a $r$-Cauchy net such that $\theta \leq f_{a} \leq u$ for all $\alpha \in\{\alpha\}, u$ is fixed in $L^{+}$. Then $f_{a} \stackrel{\hat{r}}{\rightarrow} \hat{f}$ in $\hat{L}$ for some $\hat{f}$ in $\hat{L}$. It follows that $\theta \leq \hat{f} \leq u$ and so since $L$ is an ideal of $\hat{L}$ we obtain $\hat{f}$ in $L$, i.e., $[\theta, u]$ is $r$-complete.

(ii) $\Rightarrow$ (i). Assume $\theta \leq \hat{f} \leq u, \hat{f} \in \hat{L}$ and $u \in L^{+}$. We have to show that $\hat{f}$ is in $L^{+}$, since $L$ is a Riesz subspace of $\hat{L}$. We pick a net $\left\{f_{a}\right\} \subseteq L^{+}$such that $f_{a} \stackrel{\hat{\tau}}{\rightarrow} \hat{f}$. We may suppose that $\theta \leq f_{a} \leq u$ for all $a$, otherwise we replace each $f_{a}$ by $f_{a} \wedge u$. The $r$-completeness of $[\theta, u]$ and the Hausdorff property of $r$ imply that $\hat{f}$ is in $L$, i.e., that $L$ is an ideal of $\hat{L}$.

Note. If $L$ is an ideal of $\hat{L}$ then $L$ is order dense in $\hat{L}$, i.e., $\{L\}=\hat{L}$. Indeed, if $\theta \leq \hat{f} \in L^{d}$ then $f_{a} \stackrel{\hat{\tau}}{\rightarrow} \hat{f}$ for some net $\left\{f_{a}\right\}$ of $L^{+}$, therefore $\theta=f_{a} \wedge \hat{f} \stackrel{\hat{T}}{\rightarrow} \hat{f}$, so $\hat{f}=\theta$ and hence $L^{d}=\{\theta\}$. Thus $\{L\}=L^{d d}=\hat{L}$. ( $\{D\}$ denotes the smallest band containing the nonempty subset $D_{\text {.) }}$

We recall that a net $\left\{f_{a}\right\}$ of $L$ order converges to $f$ in $L$ if there exists a net $\left\{g_{a}\right\}$ of $L$ such that $\left|f_{a}-f\right| \leq g_{a} \downarrow \theta$ in $L$. We denote this by $f_{a} \stackrel{(0)}{\longrightarrow} f$.

The next theorem deals with the embedding of $L$ into $\hat{L}$ and generalizes a result of I. Kawai (see [5, p. 296, Theorem 4.1]).

Theorem 2.3. Let $(\hat{L}, \hat{\tau})$ be the topological completion of a Hausdorff locally solid Riesz space $(L, \tau)$ and let $I: L \rightarrow \hat{L}$ be the embedding of $L$ into $\hat{L}$, i.e., $I(f)=f$ for all $f \in L$. Then the following statements are equivalent:

(i) The embedding of $L$ into $\hat{L}$, preserves arbitrary suprema and infima, or equivalently, $f_{\alpha} \downarrow \theta$ in $L$ implies $f_{\alpha} \downarrow \theta$ in $\hat{L}$. 
(ii) For every $r$-Caucby net $\left\{f_{\alpha}\right\}$ of $L^{+}$such that $f_{\alpha} \stackrel{(0)}{\longrightarrow} \theta$ in $L$, we bave that $f_{a} \stackrel{r}{\rightarrow} \theta$.

Proof. (i) $\Rightarrow$ (ii). Let $\left\{f_{a}\right\} \subseteq L^{+}$be a $r$-Cauchy net such that $f_{a} \stackrel{(0)}{\longrightarrow} \theta$ in $L$. It follows that there exists a net $\left\{g_{a}\right\} \subseteq L^{+}$such that $\theta \leq f_{a} \leq g_{a} \downarrow \theta$ in $L$. By hypothesis we have also $g_{a} \downarrow \theta$ in $\hat{L}$. We note that $\left\{f_{a}\right\}$ being a $r$-Cauchy net, is also a $\hat{r}$-Cauchy net, so $f_{\alpha} \stackrel{\hat{i}}{\rightarrow} \hat{f}$ for some $\hat{f} \in \hat{L}$. But, for fixed $\beta \in\{a\}$ and $\alpha \geq \beta$ we have $\theta \leq f_{\alpha} \leq g_{\alpha} \leq g_{\beta}$ which shows that $\theta \leq \hat{f} \leq g_{\beta}$ in $\hat{L}$ for all $\beta \in\{\alpha\}$. It follows then that $\hat{f}=\theta$, so $f_{\alpha} \stackrel{\hat{\tau}}{\rightarrow} \theta$, i.e., $f_{\alpha} \stackrel{\tau}{\rightarrow} \bar{\theta}$.

(ii) $\Rightarrow$ (i). Let $f_{\alpha} \downarrow \theta$ in $L$ and assume that $f_{\alpha} \geq \hat{f} \geq \theta$ for all $\alpha \in\{\alpha\}$ in $\hat{L}$. We have to show that $\hat{f}=\theta$. Let $\left\{g_{\lambda}\right\}$ be a net of $L^{+}$such that $g_{\lambda} \stackrel{\hat{r}}{\rightarrow} \hat{f}$. Then we have

$$
\left|g_{\lambda} \wedge f_{a}-\hat{f}\right|=\left|g_{\lambda} \wedge f_{\alpha}-\hat{f} \wedge f_{a}\right| \leq\left|g_{\lambda}-\hat{f}\right| \text { for all } \alpha, \lambda \text {. }
$$

This shows that

$$
g_{\lambda} \wedge f_{a} \frac{\hat{r}}{(\lambda, a)} \hat{f}
$$

In particular we have that $\left\{g_{\lambda} \wedge f_{a}\right\}$ is a $r$-Cauchy net of $L$. We also have $\theta \leq g_{\lambda} \wedge f_{a} \leq f_{a} \downarrow_{(\lambda, a)} \theta$ in $L$. From our hypothesis it follows that $g_{\lambda} \wedge f_{a} \frac{r}{(\lambda, a)} \theta$. Hence $\hat{f}=\theta$ and this shows that $f_{a} \downarrow \theta$ in $\hat{L}$.

Theorem 2.4. Let $(L, \tau)$ be a Hausdorff locally solid Riesz space. Then the following statements are equivalent.

(i) $L=\hat{L}$, i.e., $L$ is $r$-complete.

(ii) For every $\hat{f}, \hat{g} \in \hat{L}$ with $\hat{f}<\hat{g}$ there exists $f \in L$ sucb that $\hat{f} \leq f \leq \hat{g}$.

Theorem 2.4 is a corollary of the following general theorem.

Theorem 2.5. Let $L$ be a Riesz subspace of the Archimedean Riesz space $K$. The1 the following statements are equivalent.

(i) $L=K$.

(ii) For every $f, g \in K$ with $f<g$ there exists $u \in L$ such that $f \leq u \leq g$.

Proof. It is evident-that (i) $\Rightarrow$ (ii). We have only to show that (ii) $\Rightarrow$ (i). To this end let $\theta<u^{*} \in K$. Let $A_{u^{*}}$ be the ideal generated by $u^{*}$ in $K$. Then $L_{1}=$ $L \cap A_{u^{*}}$ is a Riesz subspace of $A_{u^{*}}$ such that for every $f^{*}, g^{*}$ in $A_{u^{*}}, f^{*}<g^{*}$ there is $u \in L_{1}$ with $f^{*} \leq u \leq g^{*}$.

By the Yosida representation theorem (see [9, Theorem 45.3, p. 308]) there exists a Hausdorff compact topological space $X$ such that $A_{u} *$ is Riesz isomorphic to a Riesz subspace $\hat{A}_{u^{*}}$ of $C(X)$, with $\hat{u}^{*}(x)=1$ for all $x \in X$ and with $\hat{A}_{u^{*}}$ separating the points of $X$. Let $V_{x_{0}}$ be an open neighborhood of the point $x_{0}$ of $X$ and let $x$ be a point of $X$ not in $V_{x_{0}}$. Then there exists a function $\hat{f}_{\epsilon} \hat{A}_{u^{*}}$ 
such that $\hat{f}(x)=2$ and $\hat{f}\left(x_{0}\right)=0$. Without loss of generality we can suppose that $\hat{f}$ is positive, otherwise we replace $\hat{f}$ by $\hat{f}^{+}$. Let $V_{x}$ be an open neighborhood of $x$ such that $\hat{f}(y)>1$ for all $y \in V_{x^{*}}$. Then the function of $\hat{A}_{u^{*}}, \hat{g}=\widehat{u^{*}} \wedge \hat{f}$, satisfies $\theta \leq \hat{g} \leq \widehat{u^{*}}=1, \hat{g}\left(x_{0}\right)=0$ and $\hat{g}(y)=1$ for all $y \in V_{x}$. Since

$$
X-V_{x_{0}} \subseteq \bigcup_{x \in X-V_{x_{0}}} V_{x} \text { and } X-V_{x_{0}} \text { is compact, }
$$

there exist neighborhoods $\left\{V_{x_{i}}: i=1, \ldots, n\right\}$ such that $X-V_{x_{0}} \subseteq \bigcup_{i=1}^{n} V_{x_{i} \cdot}$ Let

$$
\hat{g}_{0}=\bigvee_{i=1}^{n} \hat{g}_{i}=\bigvee_{i=1}^{n} g_{i}
$$

Then $\hat{g}_{0} \in \hat{A}_{u^{*}}, \hat{g}_{0}(x)=1$ for all $x$ not in $V_{x_{0}}, \hat{g}_{0}\left(x_{0}\right)=0$ and $\theta<\hat{g}_{0}<\hat{u}^{*}=1$.

Now if $X$ consists of one point then $C(X) \cong \mathbf{R}$ and from this it follows easily that $\widehat{u^{*}} \in \hat{L}$ and so $u^{*} \in L$.

Now let $X$ contain more than one point and let $x_{1}, x_{2}$ in $X$ be such that $x_{1} \neq x_{2}$. We pick two open neighborhoods $V_{x_{1}}, v_{x_{2}}$ of $x_{1}$ and $x_{2}$, respectively, such that $V_{x_{1}} \cap V_{x_{2}}=\varnothing$. Let $\hat{f}_{1}$ and $\hat{f}_{2}$ be in $\hat{A}_{u}{ }^{*}$ such that

$$
\theta \leq \hat{f}_{1}, \hat{f}_{2} \leq \widehat{u^{*}}=1, \quad \hat{f}_{1}\left(x_{1}\right)=\hat{f}_{2}\left(x_{2}\right)=0,
$$

$\hat{f}_{1}(x)=1$ for all $x$ not in $V_{x_{1}}, \hat{f}_{2}(x)=1$ for all $x$ not in $V_{x_{2}}$. Since $\hat{f}_{1}<\widehat{u}^{*}, \hat{f}_{2}<\widehat{u}^{*}$ there are $g_{1}, g_{2} \in L_{1}$, such that $\hat{f}_{1} \leq \hat{g}_{1} \leq \widehat{u}^{*}, \hat{f}_{2} \leq \hat{g}_{2} \leq \widehat{u}^{*}$. It follows from this easily that

$$
\widehat{g_{1} \vee g_{2}}=\hat{g}_{1} \vee \hat{g}_{2}=\widehat{u^{*}}=1
$$

so $\widehat{u}^{*}$ is in $\hat{L}_{1}$. Thus $u^{*} \in L_{1} \subseteq L$. This shows that $L=K$. $\square$ (See also [6, p. 273] for another proof.)

A sufficient condition for some order properties of $L$ to be inherited in $\hat{L}$ is given in the next theorem.

Theorem 2.6. Let $(L, \gamma)$ be a Hausdorff locally solid Riesz space and assume that $L$ is an ideal of $\hat{L}$. Then we have:

(i) If $L$ is $\sigma$-Dedekind complete, then $\hat{L}$ is $\sigma$-Dedekind complete.

(ii) If $L$ is Dedekind complete, then $\hat{L}$ is Dedekind complete.

Proof. The proof is similar to that of Theorem 66.5 of [7, Note XVI, p. 665].

3. The properties $(A, \mathrm{ii})$ and $(A, \mathrm{iii})$ are preserved under topological completion. The next theorem deals with property $(A, i i i)$.

Theorem 3.1. (i) The topological completion $(\hat{L}, \hat{\tau})$ of the Hausdorff locally solid 
Riesz space $(L, r)$ satisfies condition $(\Lambda$, iii) if, and only if, $(L, r)$ satisfies condition (A, iii).

(ii) If $(L, \tau)$ satisfies $(A, \mathrm{iii})$ then $\hat{L}$ is Dedekind complete and $(\hat{L}, \hat{\tau})$ satisfies (A, ii).

Proof. (i) Obviously $(L, \tau)$ satisfies condition $(\Lambda$, iii) if $(\hat{L}, \hat{\tau})$ satisfies $(A$, iii).

Now let $(L, \tau)$ satisfy $\left(A\right.$, iii) and let $\theta \leq \hat{f}_{n} \downarrow$ in $\hat{L}$. We have to show that $\left\{\hat{f}_{n}\right\}$ is a $\hat{\tau}$-Cauchy sequence. To this end let $U$ be a solid $\hat{r}$-neighborhood of zero and let $V$ be also a solid $\hat{r}$-neighborhood of zero such that $V+V+V \subseteq U$.

We construct next a sequence $\left\{V_{n}\right\}$ of solid $\hat{\tau}$-neighborhoods of zero such that $V_{1}=V$ and $V_{n+1}+V_{n+1} \subseteq V_{n}$, for $n=2,3, \ldots$. To do this start with $V_{1}=V$. Next pick a solid $\hat{r}$-neighborhood of zero $W_{1}$ such that $W_{1}+W_{1} \subseteq V$. Let $V_{2}=W_{1}$. Now pick a solid r-neighborhood of zero $W_{2}$ such that $W_{2}+W_{2} \subseteq W_{1}$. Let $V_{3}=W_{2}$. Proceeding this way we construct the above sequence $\left\{V_{n}\right\}$.

Given $n \in N$, choose an element $g_{n} \in L^{+}$such that $\left|\hat{f}_{n}-g_{n}\right| \in V_{n+1}$. Construct the sequence $\left\{f_{n}\right\}$ of $L^{+}$defined by $f_{n}=\bigwedge_{i=1}^{n} g_{i}$, for $n=1,2, \ldots$. Then it is evident that $\theta \leq f_{n} \downarrow$ in $L$. It follows then from condition $(\Lambda$, iii) on $(L, r)$ that $\left\{f_{n}\right\}$ is a $r$-Cauchy sequence and so surely it is a $\hat{r}$-Cauchy sequence. This shows in particular that $\left|f_{n}-f_{m}\right| \in V$ for all $n, m \geq n_{0}$. We also have that

$$
\begin{aligned}
\hat{f}_{n}-f_{n} & =\hat{f}_{n}-\bigwedge_{i=1}^{n} g_{i}=\bigvee_{i=1}^{n}\left(\hat{f}_{n}-g_{i}\right) \leq \bigvee_{i=1}^{n}\left(\hat{f}_{i}-g_{i}\right) \\
& \leq \sum_{i=1}^{n}\left|\hat{f}_{i}-g_{i}\right| \in V_{2}+V_{3}+\cdots+V_{n} \subset V, \\
f_{n}-\hat{f}_{n} & \leq g_{n}-\hat{f}_{n} \leq \sum_{i=1}^{n}\left|\hat{f}_{i}-g_{i}\right| \in V .
\end{aligned}
$$

So, $\left|\hat{f}_{n}-f_{n}\right| \leq \sum_{i=1}^{n}\left|\hat{f}_{i}-g_{i}\right| \in V$, i.e., $\left|\hat{f}_{n}-f_{n}\right| \in V$ for all $n=1,2, \ldots$.

Now for $n, m \geq n_{0}$ we have

$$
\left|\hat{f}_{n}-\hat{f}_{m}\right| \leq\left|\hat{f}_{n}-f_{n}\right|+\left|f_{n}-f_{m}\right|+\left|f_{m}-\hat{f}_{m}\right| \epsilon V+V+V \subseteq U
$$

Hence $\left|\hat{f}_{n}-\hat{f}_{m}\right| \in U$ for all $n, m \geq n_{0}$, which shows that $\hat{f}_{n}-\hat{f}_{m} \in U$ for all $n, m$ $\geq n_{0}$, i. e., $\left\{\hat{f}_{n}\right\}$ is a $\hat{r}$-Cauchy sequence.

(ii) Let $\theta \leq \hat{f}_{a} \uparrow \leq \hat{f}$ in $\hat{L}$. Then by (i) and Theorem 1.1(ii) we see that $\left\{\hat{f}_{a}\right\}$ is a $\hat{\tau}$-Cauchy net. Hence $\hat{f}_{a} \stackrel{\hat{\tau}}{\rightarrow} \hat{g}$ in $(\hat{L}, \hat{\tau})$. Now, Lemma 1.3 (ii) shows that $\theta \leq \hat{f_{a}} \uparrow \hat{g}$ in $\hat{L}$, i.e., $\hat{L}$ is Dedekind complete. To see that $(\hat{L}, \hat{\tau})$ satisfies $(A$, ii) observe that a complete Hausdorff locally solid Riesz space satisfies $(A, i i)$ if, and only if, it satisfies ( $\Lambda$, iii). 
Theorem 3.2. If the Hausdorff locally solid Riesz space $(L, \gamma)$ satisfies condition (A, ii), then the topological completion $(\hat{L}, \hat{\gamma})$ of $(L, r)$ satisfies also the condition ( $A, \mathrm{ii})$.

Proof. From Theorem 1.1(v) it follows that $(L, r)$ satisfies condition $(A, i i)$ and from Theorem 3.1(i) we see that $(\hat{L}, \hat{\tau})$ satisfies (A, iii). It follows now from Theorem 3.1 (ii) that $(\hat{L}, \hat{\gamma})$ satisfies $(A, i i)$.

The converse of Theorem 3.2 may be false as the following example shows.

Example 3.3. Let $L=C_{[0,1]}$ and let $r$ be the Hausdorff locally solid topology generated by the (Riesz) norm $\rho(f)=\int_{0}^{1}|f(x)| d x$. Then $L$ satisfies (A, iii) but not $(A, 0)$ (see Example 1.2(iii)) so in particular not ( $A$, ii). The completion $\hat{L}$ is the Riesz space $L_{1}([0,1])$, i.e., the Lebesgue equivalence classes with the usual ordering with $\hat{\gamma}$ generated by the (Riesz) norm $\rho([f])=\int_{0}^{1}|f(x)| d x$ and $\hat{L}^{+}=$ $\{[f]: f \geq \theta$ a.e. $\}$. It is not hard to verify that $(L, r)$ satisfies $(A, i i)$.

Generalizing the idea of the topological completion we say that the locally solid Riesz space $(\tilde{L}, \tilde{F})$ is a topological completion of the locally solid Riesz space $(L, \tau)$ (not necessarily Hausdorff) if:

(1) $(\tilde{L}, \tilde{F})$ is $\tilde{F}$-complete.

(2) $L$ is embedded in $\widetilde{L}$ as a Riesz subspace $L_{1}$ of $\tilde{L}$; we shall think of $L$ and $L_{1}$ as identical.

(3) $(L, \tau)$ is a subspace of $(\tilde{L}, \tilde{F})$, i.e., $\mathcal{F}$ induces $r$ on $L$.

(4) $L$ is $\tilde{F}$-dense in $\tilde{L}$.

The following lemma gives some information about the topological completions.

Lemma 3.4. Let $(\widetilde{L}, \widetilde{F})$ be a topological completion of the locally solid Riesz

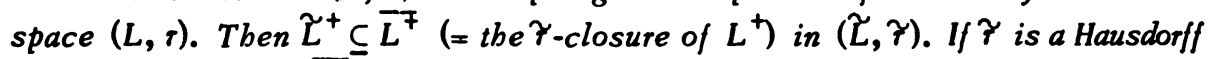
topology, then $\tilde{L}^{+}=\bar{L}^{+}$.

Proof. Let $\tilde{u} \in \tilde{L}^{+}$. Then there exists a net $\left\{u_{a}\right\}$ of $L$ such that $u_{a} \stackrel{\widetilde{q}}{\rightarrow} \tilde{u}$. Since $(\tilde{L}, \widetilde{T})$ is locally solid and $L$ is a Riesz subspace of $\widetilde{L}$ we see that $u_{\alpha}^{+} \stackrel{\tau}{\rightarrow}$ $\tilde{u}$, i.e., $\tilde{u} \in \overline{L^{7}}$. This shows that $\tilde{L}^{+} \subseteq \overline{L^{7}}$. If now $\tilde{F}$ is Hausdorff then $r$ is Hausdorff and hence $₹=\hat{\tau}$. Thus $\tilde{L}=\hat{L}$, and it follows from Theorem 2.1 that $\hat{L}^{+}=$ $\bar{L}$.

The next theorem is a generalization of Theorem 3.1(i).

Theorem 3.5. Let $(\tilde{L}, \tilde{F})$ be a topological completion of a locally solid Riesz space $(L, \tau)$. Then $(\tilde{L}, \widetilde{\tau})$ satisfies condition $(A$, iii) if, and only if, $(L, \tau)$ satisfies condition (A, iii).

Proof. The proof is similar to that of Theorem 3.1(i), taking into account Lemma 3.4. 
Next, we shall investigate under what conditions $(A, i)$ in $(L, \tau)$ implies $(A, i)$ in $(\hat{L}, \hat{\tau})$. Example 3.3 shows that $(A, i)$ in $(\hat{L}, \hat{\tau})$ does not imply $(A, i)$ in $(L, \tau)$, not even for normed Riesz spaces.

4. Upper and lower elements in the metrizable case. We shall assume next that the topology $r$ of the Hausdorff locally solid Riesz space $(L, \tau)$ is metrizable, i.e., the topology $r$ is generated by a distance, or equivalently, there exists a countable basis $\left\{V_{n}\right\}$ for the r-neighborhoods of zero such that $\bigcap_{n=1}^{\infty} V_{n}=\{\theta\}$ (see [4, Theorem 1, p. 111]). We shall call $(L, \tau)$, in this case, a metrizable locally solid Riesz space.

Given a metrizable locally solid Riesz space $(L, \tau)$ we define the following subsets of $\hat{L}$ :

$$
\begin{aligned}
& U=\left\{\hat{f} \in \hat{L}: \quad \exists\left\{f_{n}\right\} \subseteq L^{+} ; \theta \leq f_{n} \uparrow \text { and } f_{n} \stackrel{\hat{\tau}}{\rightarrow} \hat{f}\right\} \\
& \Lambda=\left\{\hat{f} \in \hat{L}: \quad \exists\left\{f_{n}\right\} \subseteq L^{+} ; \theta \leq f_{n} \downarrow \text { and } f_{n} \stackrel{\hat{\tau}}{\rightarrow} \hat{f}\right\}
\end{aligned}
$$

We shall call the elements of $U$ upper elements and the elements of $\Lambda$ lower elements.

It is easy to verify that $U$ and $\Lambda$ are cones of $\hat{L}$ and that they are closed under the lattice operations.

Concerning the metrizable locally solid Riesz spaces we have the following lemma, essentially due to W. A. J. Luxemburg [7, Theorem 60.3].

Lemma 4.1. Let $(L, \tau)$ be a metrizable locally solid Riesz space. Assume V is a r-neighborbood of zero of $\hat{L}$ and $\theta \leq \hat{f} \in \hat{L}$. Then there exists an element $\hat{u} \in U$ such that $\hat{f} \leq \hat{u}$ and $\hat{u}-\hat{f} \in V$, or in other words, every positive ele ment of $\hat{L}$ is the $\hat{r}$-limit of a decreasing sequence of upper elements.

Proof. First we pick a countable basis $\left\{V_{n}\right\}$ of solid r-closed neighborhoods of zero of $\hat{L}$ such that

$$
V_{n+1}+V_{n+1} \subseteq V_{n}, \text { for } n=1,2, \ldots
$$

Choose a $V_{k}$ from $\left\{V_{n}\right\}$. Since $\theta \leq \hat{f} \in \hat{L}$ we have $f_{n} \stackrel{\hat{\tau}}{\rightarrow} \hat{f}$ for some sequence $\left\{f_{n}\right\}$ of $L^{+}$. This implies that $\left\{f_{n}\right\}$ is a $\hat{r}$-Cauchy sequence. Let $\left\{g_{n}\right\}$ be a subsequence of $\left\{f_{n}\right\}$ such that $\left|g_{n+1}-g_{n}\right| \in V_{k+n+2}$ for $n=1,2, \cdots$, and let $u_{1}=\theta$,

$$
u_{n}=g_{n}+\sum_{i=1}^{n-1}\left|g_{i+1}-g_{i}\right| \in L^{+}, \quad n=2,3, \cdots
$$

Then $u_{n+1}-u_{n}=g_{n+1}-g_{n}+\left|g_{n+1}-g_{n}\right| \geq \theta$ for $n=2,3, \cdots$, which shows that $\theta \leq u_{n} \hat{\uparrow}$ in $L$. Further $\left\{u_{n}\right\}$ is a $\hat{\tau}$-Cauchy sequence, as we can easily verify, so $u_{n} \stackrel{\hat{\tau}}{\rightarrow} \hat{u}$ for some $\hat{u} \in \hat{L}$. Since $g_{n} \leq u_{n}$ for $n=1,2, \cdots$ and $g_{n} \stackrel{\hat{T}}{\rightarrow} \hat{f}$ in $\hat{L}$, we have also that $\hat{f} \leq \hat{u}$. 
It follows, now, from

$$
\begin{aligned}
\left|u_{n}-\hat{f}\right| & \leq\left|g_{n}-\hat{f}\right|+\sum_{i=1}^{n-1}\left|g_{i+1}-g_{i}\right| \epsilon\left|g_{n}-\hat{f}\right|+\sum_{i=1}^{n-1} v_{k+2+i} \\
& \subseteq\left|g_{n}-\hat{f}\right|+V_{k+1}
\end{aligned}
$$

that $u_{n}-\hat{f} \in V_{k}$ for all sufficiently large $n$. Since $V_{k}$ is $\hat{\tau}$-closed it follows that $\hat{u}-\hat{f} \in V_{k}$. This completes the proof of the first part.

Now, given $n \in N$ pick an upper element $\hat{f}_{n}$ such that $\hat{f}_{\leq} \hat{f}_{n}$ and $\hat{f}_{n}-\hat{f} \in V_{n}$. Let $\hat{g}_{n}=$ $\bigwedge_{i=1}^{n} \hat{f}_{i}, n=1,2, \ldots$.

Then $\hat{g}_{n}$ is an upper element for all $n, \hat{f} \leq \hat{g}_{n}$ for all $n=1,2, \ldots, \hat{g}_{n} \downarrow$ in $\hat{L}$ and $\theta \leq \hat{g}_{n}-\hat{f} \leq \hat{f}_{n}-\hat{f} \in V_{n}$, i.e., $\hat{g}_{n} \stackrel{\hat{q}}{\rightarrow} \hat{f}$. This completes the proof.

The following lemma is the "dual" of Lemma 4.1 and can be proved in a similar manner as Theorem 60.6 of [7, Note XVI, p. 649].

Lemma 4.2. Let $(L, r)$ be a metrizable locally solid Riesz space. Assume $V$ is a r-neigbborbood of zero of $\hat{L}$, and $\theta \leq \hat{f} \in \hat{L}$. Then there exists an element $\hat{u} \in \Lambda$, such that $\hat{u} \leq \hat{f}$ and $\hat{f}-\hat{u} \in V$, or in other words, every positive element of $\hat{\boldsymbol{L}}$ is the $\hat{\tau}$-limit of an increasing sequence of lower elements.

5. The properties $(A, i)$ and $(B, i)$ in the metrizable case. We prove first that the property $(\mathrm{A}, \mathrm{i})$ is preserved under topological completion of the metrizable locally solid Riesz spaces.

Theorem 5.1. The completion $(\hat{L}, \hat{\gamma})$ of the metrizable locally solid Riesz space $(L, r)$ satisfies $(A, i)$ if $(L, r)$ satisfies $(A, i)$.

Proof. Let $\left\{V_{n}\right\}$ be a countable basis of the neighborhood system of the origin of $\hat{L}$ consisting of solid sets and let $\hat{f}_{n} \downarrow \theta$ in $\hat{L}$. We shall show that $\hat{f}_{n} \stackrel{\hat{q}}{\rightarrow} \theta$.

For a given $n \in N$, let $\hat{u}_{n} \in U$ be such that $\theta \leq \hat{f}_{n} \leq \hat{u}_{n}$ and $\hat{u}_{n}-\hat{f}_{n} \in V_{n}$. This is possible by Lemma 4.1. Let $\hat{w}_{n}=\bigwedge_{i=1}^{n} \hat{u}_{i} \in U$, then we have $\theta \leq \hat{f}_{n} \downarrow \leq$ $\hat{w}_{n} \downarrow$ in $\hat{L}$ and $\theta \leq \hat{w}_{n}-f_{n} \leq \hat{u}_{n}-\hat{f}_{n} \in V_{n}$, for $n=1, \ldots$, so $\hat{w}_{n}-\hat{f}_{n} \stackrel{\tau}{\rightarrow} \theta$.

Since $\hat{f}_{n} \downarrow \theta$ in $\hat{L}$ it follows from Lemma 1.4 that $\hat{w}_{n} \downarrow \theta$ in $\hat{L}$. This shows that we may assume that $\left\{\hat{f}_{n}\right\} \subseteq U$, i.e., that $\left\{\hat{f}_{n}\right\}$ is a sequence of upper elements.

Now let $V_{k}$ be one neighborhood from the sequence $\left\{V_{n}\right\}$. Given $n \in N$, choose an element $g_{n} \in L^{+}$such that $\hat{f}_{n}-g_{n} \in V_{k+n+2}$, and $\theta \leq g_{n} \leq \hat{f}_{n}$. Note that this is possible since $\left\{\hat{f}_{n}\right\} \subseteq U$.

Let $f_{n}=\bigwedge_{i=1}^{n} g_{i} \in L^{+}$for $n=1,2, \ldots$. Then we have

$$
\begin{aligned}
\theta & \leq \hat{f}_{n}-f_{n}=\hat{f}_{n}-\bigwedge_{i=1}^{n} g_{i}=\bigvee_{i=1}^{n}\left(\hat{f}_{n}-g_{i}\right) \leq \bigwedge_{i=1}^{n}\left(\hat{f}_{i}-g_{i}\right) \\
& \leq \sum_{i=1}^{n}\left(\hat{f}_{i}-g_{i}\right) \in V_{k+3}+\cdots+V_{k+n+2} \subseteq V_{k+1} .
\end{aligned}
$$


So, $\hat{f}_{n}-f_{n} \in V_{k+1}$ for $n=1,2, \ldots$.

Now we observe that $f_{n} \downarrow \theta$ in $L$, so $f_{n} \stackrel{\tau}{\rightarrow} \theta$ since $(A, i)$ holds in $(L, r)$. This implies that $f_{n} \stackrel{\hat{r}}{\rightarrow} \theta$. In particular we have $f_{n} \in V_{k+1}$ for all $n \geq n_{0}$. So, we have $\hat{f}_{n}=f_{n}+\left(\hat{f}_{n}-f_{n}\right) \in V_{k+1}+V_{k+1} \subseteq V_{k}$ for $n \geq n_{0}$, hence $\hat{f}_{n} \in V_{k}$ for $n \geq n_{0}$, i.e., $\hat{f}_{n} \stackrel{\hat{\imath}}{\rightarrow} \theta$.

We recall that a positive operator $T$ from a Riesz space $L$ into another is called an integral if $u_{n} \downarrow \theta$ in $L$ implies $T\left(u_{n}\right) \downarrow \theta$ in $M$.

Theorem 5.2. Assume that $(L, \tau)$ and $(M, \sigma)$ are two Hausdorff locally solid Riesz spaces with $(L, r)$ metrizable and with $(M, \sigma)$ satisfying $(A, i)$.

If $T:(L, \tau) \rightarrow(M, \sigma)$ is a positive continuous linear operator which is also an integral, then the unique linear continuous extension $\hat{T}:(\hat{L}, \hat{\tau}) \rightarrow(\hat{M}, \hat{\sigma})$ is also an integral.

Proof. Obviously the continuous linear extension of $T, \hat{T}$, is positive. Let $\hat{u}_{n} \downarrow \theta$ in $\hat{L}$. We have to show that $\hat{T}\left(\hat{u}_{n}\right) \downarrow \theta$ in $\hat{M}$. We can assume that $\left\{\hat{u}_{n}\right\} \subseteq U$. Indeed, as in the previous theorem we can construct a decreasing sequence $\left\{\hat{w}_{n}\right\} \subseteq U$ such that $\theta \leq \hat{u}_{n} \leq \hat{w}_{n}$ for $n=1,2, \ldots$ and $\hat{w}_{n}-\hat{u}_{n} \stackrel{\hat{q}}{\rightarrow} \theta$. So, $\theta \leq \hat{T}\left(\hat{u}_{n}\right) \downarrow \leq \hat{T}\left(\hat{w}_{n}\right) \downarrow$ for $n=1,2, \ldots$ and $\theta \leq \hat{T}\left(\hat{w}_{n}\right)-\hat{T}\left(\hat{u}_{n}\right)=\hat{T}\left(\hat{u}_{n}^{n}-\hat{w}_{n}^{n}\right) \stackrel{\hat{\sigma}}{\rightarrow} \theta$. Using Lemma 1.4 we can see that our assertion is valid. So, let $\left\{\hat{u}_{n}\right\} \subseteq U$ be such that $\hat{u}_{n} \downarrow \theta$ in $\hat{L}$. We have to show that $\hat{T}\left(\hat{u}_{n}\right) \downarrow \theta$ in $\hat{M}$. Assume that $\hat{T}\left(\hat{u}_{n}\right) \geq \hat{b} \geq \theta$ for $n=1,2, \ldots$ and some $\hat{b} \in \hat{M}$.

Let $W$ be a solid $\hat{\sigma}$-neighborhood of zero of $\hat{M}$ and let $W_{1}$ be another $\hat{\sigma}-n$ eighborhood of zero of $\hat{M}$ such that $W_{1}+W_{1} \subseteq W$. We choose next a solid $\hat{f}$-neighborhood $V$ of zero of $\hat{L}$ such that $\hat{T}(\hat{u}) \in W_{1}$ for all $\hat{u} \in V$. Let $\left\{V_{n}\right\}$ be a basis for the neighborhood system of the origin of $\hat{L}$ consisting of solid sets and such that $V_{n+1}$ $+V_{n+1} \subseteq V_{n}, n=1,2, \cdots$ and $V_{1}+V_{1} \subseteq V$.

Given $n \in N$ pick an element $\theta \leq u_{n} \in L^{+}$such that $\theta \leq u_{n} \leq \hat{u}_{n}$ and $\theta \leq \hat{u}_{n}-$ $u_{n} \in V_{n}$. Let $w_{n}=\bigwedge_{i}^{n} u_{i}, n=1,2, \ldots$. Then obviously $w_{n} \downarrow \theta$ in $L$, and so $T\left(w_{n}\right) \stackrel{\downarrow}{\downarrow} \theta$ in $M$. Hence it follows from $(A, i)$ of $(M, \sigma)$ that $T\left(w_{n}\right) \stackrel{\sigma}{\rightarrow} \theta$. So in particular $T\left(w_{n}\right) \in W_{1}$ for all $n \geq n_{0}$.

We observe now that

$$
\begin{aligned}
\theta & \leq \hat{u}_{n}-w_{n}=\hat{u}_{n}-\bigwedge_{i=1}^{n} u_{i}=\bigvee_{i=1}^{n}\left(\hat{u}_{n}-u_{i}\right) \leq \bigvee_{i=1}^{n}\left(\hat{u}_{i}-u_{i}\right) \\
& \leq \sum_{i=1}^{n}\left(\hat{u}_{i}-u_{i}\right) \in V_{1}+\cdots+V_{n} \subseteq V_{1}+V_{1} \subseteq V,
\end{aligned}
$$

so $\hat{u}_{n}-w_{n} \in V$ for $n=1,2, \ldots$. Hence $\theta \leq \hat{b} \leq \hat{T}\left(\hat{u}_{n}\right)=\hat{T}\left(\hat{u}_{n}-w_{n}\right)+T\left(w_{n}\right) \in W_{1}+$ $W_{1} \subseteq W$ for all $n \geq n_{0}$. So $\hat{b} \in W$ for all $\hat{\sigma}$-neighborhoods $W$ of zero of $\hat{M}$. Since $\hat{\sigma}$ is a Hausdorff topology we have $\hat{b}=\theta$, i.e., $\hat{T}\left(\hat{u}_{n}\right) \downarrow \theta$. This completes the proof. 
The following theorem says that $(B, i)$ is preserved under the topological completion of a metrizable locally solid Riesz space.

Theorem 5.3. The topological completion $(\hat{L}, \hat{\gamma})$ of a metrizable locally solid Riesz space $(L, r)$ satisfies $(B, i)$ if, and only if, $(L, \tau)$ satisfies $(B, i)$.

Proof. Obviously if $(\hat{L}, \hat{\tau})$ satisfies $(B, i)$ then $(L, \tau)$ satisfies $(B, i)$. So assume $(L, \tau)$ satisfies $(B, i)$ and let $\theta \leq \hat{f}_{n} \uparrow$ be a $\hat{\tau}$-bounded sequence of $\hat{L}$. We have to show that $\left\{\hat{f}_{n}\right\}$ is a $\hat{\tau}$-Cauchy sequence. To do this, let $\left\{V_{n}\right\}$ be a sequence of solid $\hat{\gamma}$-neighborhoods of zero such that $V_{n+1}+V_{n+1} \subseteq V_{n}$ for $n=1,2, \ldots$. Pick a $V_{k}$ from $\left\{V_{n}\right\}$. Given $n>k+2$ choose an element $g_{n} \in L^{+}$such that $\mid \hat{f}_{n}-$ $g_{n} \mid \in V_{n+1}$, and construct the sequence $f_{n}=\bigvee_{i=k+3}^{n} g_{i}$ for $n=k+3, k+4, \cdots$ Then we have $\theta \leq f_{n} \uparrow$ in $L$, and

$$
\begin{aligned}
f_{n}-\hat{f}_{n} & =\bigvee_{i=k+3}^{n}\left(g_{i}-\hat{f}_{n}\right) \leq \bigvee_{i=k+3}^{n}\left(g_{i}-\hat{f}_{i}\right) \\
& \leq \sum_{i=k+3}^{n}\left|g_{i}-\hat{f}_{i}\right| \in V_{k+3}+\cdots+V_{n} \subseteq V_{k+2}, \\
\hat{f}_{n}-f_{n} & \leq \hat{f}_{n}-g_{n} \leq \sum_{i=k+3}^{n}\left|g_{i}-\hat{f}_{i}\right|,
\end{aligned}
$$

for all $n>k+2$, so, $\left|\hat{f}_{n}-f_{n}\right| \leq \Sigma_{i=k+3}^{n}\left|\hat{f}_{i}-g_{i}\right| \in V_{k+2}$; hence $\left|f_{n}-\hat{f}_{n}\right| \in V_{k+2}$ for $n=k+3, k+4, \cdots$.

On the other hand we have

$$
\theta \leq \sum_{i=k+3}^{n}\left|\hat{f}_{i}-g_{i}\right| \uparrow \text { and }\left\{\sum_{i=k+3}^{n}\left|\hat{f}_{i}-g_{i}\right|: n \geq k+3\right\}
$$

is a $\hat{\gamma}$-Cauchy sequence. Hence

$$
\sum_{i=k+3}^{n}\left|\hat{f}_{i}-g_{i}\right| \frac{\hat{\imath}}{n \geq k+3, n \rightarrow+\infty} \hat{f}
$$

for some $\hat{f} \in \hat{L}$. It follows from Lemma $1.3\left(\right.$ ii) that $\Sigma_{i=k+3}^{n}\left|\hat{f}_{i}-g_{i}\right| \uparrow \hat{f}$, so, $\theta \leq$ $\left|\hat{f}_{n}-f_{n}\right| \leq \hat{f}$, for $n=k+3, k+4, \ldots$. Since $[\theta, \hat{f}]$ is $\hat{\tau}$-bounded $\left\{\hat{f}_{n}-f_{n}: n \geq \hat{k}+3\right\}$ is $\hat{\mathrm{i}}$-bounded. This implies that $\left\{\hat{f}_{n}-f_{n}\right\}$ is $\hat{\mathrm{r}}$-bounded.

We observe now that $f_{n}=\hat{f}_{n}-\left(\hat{f}_{n}-f_{n}\right)$ for $n=1,2, \ldots$ and by hypothesis $\left\{\hat{f}_{n}\right\}$ is a $\hat{\tau}$-bounded sequence. Hence $\left\{f_{n}\right\}$ is a $\hat{\tau}$-bounded sequence of $\hat{L}$ and so surely it is a $r$-bounded sequence of $L$. Now, since $(L, r)$ satisfies $(B, i)\left\{f_{n}\right\}$ is a $r$-Cauchy sequence of $L$ and so it is also a $\hat{r}$-Cauchy sequence of $\hat{L}$.

This implies in particular that $\left|f_{n}-f_{m}\right| \in V_{k+2}$ for all $n, m \geq n_{0}>k+3$.

Now, for $n, m \geq n_{0}$ we have 


$$
\left|\hat{f}_{n}-\hat{f}_{m}\right| \leq\left|\hat{f}_{n}-f_{n}\right|+\left|f_{n}-f_{m}\right|+\left|f_{m}-\hat{f}_{m}\right| \in V_{k+2}+V_{k+2}+V_{k+2} \subseteq V_{k},
$$

so, $\hat{f}_{n}-\hat{f}_{m} \in V_{k}$ for all $n, m \geq n_{0}$, i.e., $\left\{\hat{f}_{n}\right\}$ is a $\hat{f}$-Cauchy sequence.

6. The property $(A, 0)$ in the metrizable case. We recall that a locally solid Riesz space $(L, \tau)$ satisfies condition $(A, 0)$ whenever it follows from $u_{n} \downarrow \theta$ in $L$ and $\left\{u_{n}\right\}$ a $r$-Cauchy sequence that $u_{n} \stackrel{\tau}{\rightarrow} \theta$. It is evident that if $(L, \tau)$ is $r$-complete and Hausdorff then $(L, \tau)$ satisfies the property $(A, 0)$.

The following theorem gives some characterizations of the $(A, 0)$ condition for the metrizable locally solid Riesz spaces and generalizes a result of W. A. J. Luxemburg [7, Theorem 61.5, p. 652].

Theorem 6.1. Let $(L, r)$ be a metrizable locally solid Riesz space. Then the following conditions are equivalent:

(i) L satisfies condition $(A, 0)$.

(ii) For every $\theta<\hat{f} \in \hat{L}$ there exists $f \in L$ such tbat $\theta<f \leq \hat{f}$.

(iii) For every $\theta \leq \hat{f} \in \hat{L}$ we have $\hat{f}=\sup \{f \in L: \theta \leq f \leq \hat{f}\}$.

(iv) The embedding of $L$ into $\hat{L}$ preserves arbitrary suprema and infima, i.e., $f_{a} \downarrow \theta$ in $L$ implies $f_{\alpha} \downarrow \theta$ in $\hat{L}$.

(v) The embedding of $L$ into $\hat{L}$ preserves countable infima and countable suprema, i.e., $f_{n} \downarrow \theta$ in $L$ implies $f_{n} \downarrow \theta$ in $\hat{L}$.

Note. Condition (iii) is expressed by saying that $L$ is order dense in $\hat{L}$.

Proof. (ii) $\Leftrightarrow$ (iii). Suppose that (iii) holds and $\theta<\hat{f} \in \hat{L}$. Since $\hat{f}=$ $\sup \{f \in L: \theta \leq f \leq \hat{f}\}$ in $\hat{L}$ it follows that $\theta<f \leq \hat{f}$ for some $f$ in $L$ and this shows that (ii) holds. Suppose now that (ii) holds, and $\theta \leq \hat{f} \in \hat{L}$. Let $\hat{g} \in \hat{L}$ be such that $f \in L ; \theta \leq f \leq \hat{f}$ implies $f \leq \hat{g}$. Assume further that $\hat{g} \leq \hat{f}$. If $\hat{g}<\hat{f}$, then since (ii) holds, we have $\theta<f \leq \hat{f}-\hat{g} \leq \hat{f}$ for some $\theta<f \in L$. This also implies, according to our assumptions, that $\theta<f \leq \hat{g}$ and so $\theta<2 f \leq \hat{f}$ which also implies $\theta \leq$ $2 f \leq \hat{g}$. Proceeding inductively we see that $\theta<n f \leq \hat{f}$ for $n=1,2, \ldots$ and $\theta<f$. This contradicts the fact that $\hat{L}$ is Archimedean (Lemma 1.3(i)). So $\hat{g}=\hat{f}$ and this shows that $\hat{f}=\sup \{f \in L: \theta \leq f \leq \hat{f}\}$.

(iii) $\Rightarrow$ (iv). Let $f_{a} \downarrow \theta$ in $L$. If $\theta<\hat{f} \leq f_{a}$ for all $a \in\{a\}$ holds in $\hat{L}$, then since (ii) $\Leftrightarrow$ (iii) there exists an element $\theta<f \in L$ such that $\theta<f \leq \hat{f} \leq f_{a}$ for all $a \in\{a\}$, i.e., $\inf \left\{f_{\alpha}\right\} \neq \theta$ in $L$, a contradiction. This shows that $f_{a} \downarrow \theta$ in $\hat{L}$.

(iv) $\Rightarrow$ (v). Obvious.

(v) $\Rightarrow$ (i). Let $f_{n} \downarrow \theta$ in $L$ and $\left\{f_{n}\right\}$ be a r-Cauchy sequence. Then $f_{n} \stackrel{\hat{\tau}}{\rightarrow} \hat{f}$ in $\hat{L}$ for some $\hat{f} \in \hat{L}$, and so by Lemma 1.3(ii) we have $f_{n} \downarrow \hat{f}$ in $\hat{L}$. Since (v) holds we get $\hat{f}=\theta$, i.e., $f_{n} \stackrel{\tau}{\rightarrow} \theta$.

(i) $\Rightarrow$ (ii). Let $\theta \leq \hat{f} \in \hat{L}$ and assume that if $f \in L$ with $\theta \leq f \leq \hat{f}$, then $f=\theta$. We show that this assumption implies $\hat{f}=\theta$. To this end, let $V$ be a solid $\hat{\tau}$-neighborhood of zero in $\hat{L}$. By Lemma 4.2 there exists $\hat{g} \in \Lambda$ (a lower element) such 
that $\theta \leq \hat{g} \leq \hat{f}$ and $\hat{f}-\hat{g} \in V$. So there exists a sequence $\left.\lg _{n}\right\} \subseteq L^{+}$such that $\hat{g} \leq g_{n} \downarrow$ and $g_{n} \stackrel{\hat{q}}{\rightarrow} \hat{g}$, so in particular $\left\{g_{n}\right\}$ is a $r$-Cauchy sequence. We show next that $g_{n} \downarrow \theta$ in $L$. Indeed, if $\theta \leq 8 \leq g_{n}$ for all $n$, then it follows that $\theta \leq 8$ $\leq \hat{g} \leq \hat{f}$ and so by our hypothesis $g=\theta$, i.e., $g_{n} \downarrow \theta$ in $L$. Hence, by property $(\Lambda, 0)$ of $(L, r)$ it follows that $g_{n} \stackrel{r}{\rightarrow} \theta$ in $L$ and so $g_{n} \stackrel{\hat{r}}{\rightarrow} \theta$ in $\hat{L}$. This implies that $\hat{g}=\theta$. But then, $\hat{f}=\hat{f}-\theta=\hat{f}-\hat{g} \in V$; for all neighborhoods $V$ of the origin of $\hat{L}$ and this implies that $\hat{f}=\theta$. This completes the proof.

Corollary 6.2. If a metrizable locally solid Riesz space $(L, \gamma)$ satisfies $\hat{L}^{+}=$ $U$, i.e., if every positive element of $\hat{L}$ is an upper element, then $(L, \gamma)$ satisfies condition $(\mathrm{A}, \mathbf{0})$.

Proof. Let $\theta \leq \hat{f} \in \hat{L}$. Then we have $\theta \leq f_{n} \uparrow \hat{f}$ for some sequence $\left\{f_{n}\right\} \subseteq L^{+}$ with $f_{n} \stackrel{\hat{q}}{\rightarrow} \hat{f}$. So in particular we have $\hat{f}=\sup \{f \in L: \theta \leq f \leq \hat{f}\}$. It follows now from Theorem 6.1 that $(L, r)$ satisfies $(\Lambda, 0)$.

Next we shall investigate under which conditions the Dedekind completeness, $\sigma$-Dedekind completeness and super Dedekind completeness of $L$ can be carried to the topological completion. We proceed with the following lemma.

Lemma 6.3. If a metrizable locally solid Riesz space $(L, \tau)$ satisfies condition $(\mathrm{A}, 0)$ and if $L$ is $\sigma$-Dedekind complete, then we bave:

(i) Every interval of $L$ is r-complete, i.e., $[\theta, u]$ is r-complete for every $u \in L^{+}$.

(ii) $\hat{L}^{+}=U$, i.e., every positive element of $\hat{L}$ is an upper element.

Proof. Let $\hat{f} \in \Lambda$, i.e., let $\hat{f}$ be a lower element. This means there exists a sequence $\left\{f_{n}\right\} \subseteq L^{+}$such that $f_{n} \downarrow \hat{f}$ and $f_{n} \stackrel{\hat{T}}{\rightarrow} \hat{f}$. So in particular $\left\{f_{n}\right\}$ is a $r$-Cauchy sequence. Since $\theta \leq f_{n} \downarrow$ in $L$ and $L$ is $\sigma$-Dedekind complete we have $f_{n} \downarrow f \in L^{+}$in $L$ for some $f$. But then it follows from Theorem 6.1 that $\hat{f}=f \in L^{+}$. So, $\Lambda \subseteq L^{+}$, i.e., the lower elements of $\hat{L}$ are the positive elements of $L$.

(i) Now let $\theta \leq \hat{f} \leq f$ and $f \in L$. From Lemma 4.2 and the above discussion it follows that there exists a sequence $\left\{f_{n}\right\} \subseteq L^{+}, f_{n} \uparrow$ and $f_{n} \stackrel{\hat{q}}{\rightarrow} \hat{f}$. But then $f_{n} \uparrow$ $f_{0} \leq f$ in $L$, so $f_{0}-f_{n} \downarrow \theta$ in $L$ and $\left\{f_{0}-f_{n}\right\}$ is a $r$-Cauchy sequence. Hence from condition $(A, 0)$ of $(L, r)$ we see that $f_{n} \stackrel{\hat{r}}{\rightarrow} f$, so $\hat{f}=f \in L$. This shows that $L$ is an ideal of $\hat{L}$. The result of (i) now follows immediately from Theorem 2.2.

(ii) We only have to show that $\hat{f} \in \hat{L}^{+}$implies $\hat{f} \in U$. This is a direct applition of Lemma 4.2 and the above discussion.

Next we give an application of Lemma 6.3.

We recall that a subset $A$ of a Riesz space $L$ is called order complete whenever it follows from $f_{a} \uparrow \leq f$ in $L$ and $\left\{f_{a}\right\} \subseteq A$ that $\sup \left\{f_{a}\right\}$ exists in $L$ and $\sup \left\{f_{a}\right\} \in A$. A locally solid Riesz space $(L, r)$ is called a locally order complete Riesz space if there is a neighborhood basis of zero for $r$ consisting of solid and order complete sets. (See [10, p. 139].) 
With respect to the above notion we have the following theorem due to H. Nakano (see [13]).

Theorem 6.4. If $(L, r)$ is a locally order complete Riesz space, then every order interval is r-complete.

We use Lemma 6.3 to give a different proof of Theorem 6.4 in the case in which $r$ is metrizable. It is evident that $L$ is Dedekind complete and so in particular it is $\sigma$-Dedekind complete. We prove next that $(\Lambda, 0)$ is satisfied in $(L, r)$. Indeed, if $\left\{f_{n}\right\}$ is a $r$-Cauchy sequence of $L$ and $f_{n} \downarrow \theta$ in $L$ then we have $f_{n}-f_{m}$ $\epsilon V$ for all $n, m \geq n_{0}$, where $V$ is a solid and order complete neighborhood of zero. So for fixed $n \geq n_{0}$, we have $f_{n}-f_{m} \uparrow_{m \geq n_{0}} f_{n}$ in $L$, thus $f_{n} \in V$ for all $n \geq n_{0}$ and this shows that $f_{n} \stackrel{r}{\rightarrow} \theta$, i.e., $(\Lambda, 0)$ is satisfied in $(L, \tau)$. The result now follows immediately from Lemma 6.3 .

Theorem 6.5. Let $(L, r)$ be a metrizable locally solid Riesz space satisfying condition $(\mathrm{A}, 0)$.

(i) If $L$ is $\sigma$-Dedekind complete, then $\hat{L}$ is $\sigma$-Dedekind complete.

(ii) If $L$ is Dedekind complete, then $\hat{L}$ is Dedekind complete.

(iii) If $L$ is super Dedekind complete, then $\hat{L}$ is super Dedekind complete.

Proof. From Lemma 6.3(i) it follows that $L$ is an ideal of $\hat{L}$. So, (i) and (ii) follow immediately from Theorem 2.6.

(iii) It follows from Lemma 6.3 that $L$ is a super order dense ideal of $\hat{L}$ which by its own right is a super Dedekind complete Riesz space. Also by (i) we have that $\hat{L}$ is a $\sigma$-Dedekind complete Riesz space. The result now follows from Theorem 29.5 of [9, p. 169].

Note. The condition $(A, 0)$ is essential for Theorem 6.5. In [7, p. 655, Example 66.6], W. A. J. Luxemburg exhibits a super Dedekind complete normed Riesz space $L_{\rho}$ whose norm completion is not even $\sigma$-Dedekind complete.

We continue this section by generalizing a result of Luxemburg and Zaanen [8, Theorem 33.8, Note X, p. 505], and one of Nakano [11, pp. 321-322].

Theorem 6.6. Let $(L, \gamma)$ be a metrizable locally solid Riesz space. Then the following statements are equivalent:

(i) $(L, r)$ satisfies $(\mathrm{A}, \mathrm{ii})$.

(ii) $(L, r)$ satisfies botb $(\Lambda, 0)$ and $(\Lambda$, iii).

(iii) $(L, r)$ satisfies botb $(\Lambda, i)$ and $(A, i i i)$.

Proof. Obviously (ii) holds if, and only if, (iii) holds. Also it follows from Theorem 1.1(v) that (i) implies (ii). So we have to show only that (ii) implies (i). To this end let, $\theta \leq u_{a} \uparrow u$ in $L$. It follows from Theorem 1.1(ii) that $\left\{u_{a}\right\}$ is a r-Cauchy net. So $u_{a} \stackrel{\hat{q}}{\rightarrow} \hat{u}$ for some $\hat{u}$ in $\hat{L}$. But then by Lemma 1.3(ii) 
we have $u_{a} \uparrow \hat{u}$ in $\hat{L}$. It follows now from Theorem 6.1 that $u=\hat{u}$, i.e., $u_{a} \stackrel{\tau}{\rightarrow} u$ and this shows that $(L, \tau)$ satisfies the property $(A, i i)$.

Theorem 6.7. Let $(L, r)$ be a metrizable locally solid Riesz space. Then the following statements are equivalent:

(i) $(L, \gamma)$ is $\sigma$-Dedekind complete and $(\mathrm{A}, \mathrm{i})$ bolds.

(ii) $(L, \tau)$ is super Dedekind complete and $(\mathrm{A}, \mathrm{ii})$ bolds.

Proof. Obviously (ii) implies (i). We show next that (i) implies (ii). We see easily that if (i) holds then the $(A, i i i)$ condition also holds in $(L, \tau)$. Hence from Theorem 6.6 we obtain that $(A, i i)$ holds in $(L, r)$.

Now let $\theta \leq u_{a} \uparrow \leq u_{0}$ in $L$. It follows that $\left\{u_{a}\right\}$ is a $r$-Cauchy net. Hence $u_{\alpha} \stackrel{\hat{q}}{\rightarrow} \hat{u}$ for some $\hat{u} \in \hat{L}$. Since $\hat{\tau}$ is metrizable there exists a sequence $\left\{u_{\alpha_{n}}\right\} \subseteq$ $\left\{u_{a}\right\}$ such that $u_{a_{n}} \stackrel{\hat{I}}{\rightarrow} \hat{u}$. We can suppose that $\theta \leq u_{a_{n}} \uparrow$. It follows from the $\sigma-$ Dedekind completeness of $L$ that $u_{a_{n}} \uparrow u$ for some $u \in L$. Combining Lemma 1.3(ii) and Theorem 6.1 we see that $\theta \leq u_{\alpha} \uparrow u$ and this shows that $L$ is super Dedekind complete.

The Example 1.2(v) shows that Theorems 6.6 and 6.7 can be false for nonmetrizable topologies.

From Theorems 3.1(ii) and 6.7 the following result should be clear now.

Theorem 6.8. Let $(L, \tau)$ be a metrizable locally solid Riesz space. If $(L, \tau)$ satisfies $(A$, iii) then $\hat{L}$ is super Dedekind complete and $(\hat{L}, \hat{\tau})$ satisfies $(A, i i)$.

7. The projection properties. We start with the following lemma generalizing a theorem of W. A. J. Luxemburg [7, Note XVI, Theorem 66.3, p. 664].

Lemma 7.1. Let $(L, \tau)$ be a Hausdorff locally solid Riesz space. Then the closure $\bar{A}$ in $(\hat{L}, \hat{\gamma})$ of the projection band $A$ of $L$ is a projection band of $\hat{L}$. Moreover $\overline{A^{d}}=(\bar{A})^{d}$.

Proof. Let $A$ be a projection band of $L$. Then $L=A \oplus A^{d}$. We will show that $\hat{L}=\bar{A} \oplus \overline{A^{d}}$, where - denotes the closure in $(\hat{L}, \hat{\tau})$. So let $\theta \leq \hat{f} \in \hat{L}$. Then there exists a net $\left\{f_{a}\right\} \subseteq L^{+}$such that $f_{a} \stackrel{\hat{\tau}}{\rightarrow} \hat{f}$. But then since $L=A \oplus A^{d}$ we can write $f_{a}=f_{a}^{\prime}+f_{a}^{\prime \prime}, \theta \leq f_{a}^{\prime} \in A, \theta \leq f_{a}^{\prime \prime} \in A^{d}$ for all $a \in\{a\}$, and so $\mid f_{a_{1}}-$ $f_{a_{2}}|=| f_{a_{1}}^{\prime}-f_{a_{2}}^{\prime}|+| f_{a_{1}}^{\prime \prime}-f_{a_{2}}^{\prime \prime} \mid$ for all $a_{1}, a_{2}$ (see [9, Theorem 14.4(i), p. 69]).

The last relation shows that the nets $\left\{f_{a}^{\prime}\right\}$ and $\left\{f_{a}^{\prime \prime}\right\}$ are two $\hat{\gamma}$-Cauchy nets of $\hat{L}$. So $f_{a}^{\prime} \stackrel{\hat{\tau}}{\rightarrow} \hat{f}_{1}$ and $f_{a}^{\prime \prime} \stackrel{\hat{\tau}}{\rightarrow} \hat{f}_{2}$ for some $\hat{f}_{1}, \hat{f}_{2} \in \hat{L}$. So $\hat{f}=\hat{f}_{1}+\hat{f}_{2} \in \bar{A}+\overline{A^{d}}$, i.e., $\hat{L}=\bar{A}+\overline{A^{d}}$. To show that $\bar{A} \cap \overline{A^{d}}=\{\theta\}$, let $\theta \leq \hat{f} \in \bar{A} \cap \overline{A^{d}}$. Then there are two nets of $L^{+},\left\{f_{a}\right\} \subseteq A$ and $\left\{g_{a}\right\} \subseteq A^{d}$ such that $f_{\alpha} \stackrel{\hat{q}}{\rightarrow} \hat{f}$ and $g_{a} \stackrel{\hat{q}}{\rightarrow} \hat{f}$. Therefore $\theta=$ $f_{a} \wedge g_{a} \stackrel{\hat{f}}{\rightarrow} \hat{f} \wedge \hat{f}=\hat{f}$; hence $\hat{f}=\theta$ and this shows that $\hat{L}=\bar{A} \oplus \overline{A^{d}}$. We show next that $\bar{A}$ and $A^{d}$ are ideals of $\hat{L}$. It is clear that $\bar{A}$ is a vector subspace of $\hat{L}$. Now 
let $\hat{f} \in \bar{A}$. Then $f_{a} \stackrel{\hat{\tau}}{\rightarrow} \hat{f}$ for some net $\left\{f_{a}\right\} \subseteq A$. But then $\left\{\left|f_{a}\right|\right\} \subseteq A$ and $\left|f_{a}\right| \stackrel{\hat{\tau}}{\rightarrow}$ $|\hat{f}|$, so $|\hat{f}| \in \bar{A}$. Now if $\theta \leq \hat{f} \leq \hat{g}$ and $\hat{g} \in \bar{A}$ it follows easily that $\hat{f} \in \bar{A}$. This shows that $\bar{A}$ is an ideal of $\hat{L}$. Similarly for $A^{d}$. The conclusion now follows from Theorem 24.1 of $[9$, p. 131].

An element $\theta<e$ of a Riesz space $L$ is called a weak unit if $B_{e}=L$, i.e., if the band generated by $e$ is all of $L$. Note that for Archimedean Riesz spaces $\theta<e$ is a weak unit if, and only if, $|f| \wedge e=\theta$ implies $f=\theta$.

A condition is given next in order for $\hat{L}$ to possess a weak unit.

Theorem 7.2. Let $(\hat{L}, \hat{\tau})$ be a Hausdorff locally solid Riesz space with a weak unit. Assume that $L$ is order dense in $\hat{L}$. Then $\hat{L}$ bas a weak unit.

Proof. Let $\theta<e \in L$ be a weak unit of $L$ and let $\theta \leq \hat{f} \in \hat{L} ; \hat{f} \wedge e=\theta$. Assume $\theta<\hat{f}$. Pick an element $\theta<f \in L$ such that $\theta<f \leq \hat{f}$. But then $f \wedge e=\theta$, a contradiction. Hence $\hat{f}=\theta$ and so $B_{e}=\hat{L}$, i.e., $e$ is a weak unit of $\hat{L}$.

Combining the above two results we get the following:

Corollary 7.3. Let $(L, \tau)$ be a Hausdorff locally solid Riesz space which is order dense in $\hat{L}$ (in particular if $r$ is metrizable with $(A, 0)$ ). If $\theta<u \in L$ and if the band $B_{u}$ generated by $u$ in $L$ is a projection band, then the closure of $B_{u}$ in $(L, \gamma)$ is the band generated in $\hat{L}$ by $u$.

Proof. Since $u$ is a weak unit of $B_{u}$, Theorem 7.2 implies that $u$ is a weak unit of $\bar{B}_{u}$ (the closure of $B_{u}$ in $\hat{L}$ ). By Lemma 7.1, $\bar{B}_{u}$ is a band of $\hat{L}$ and by Lemma 1.3 (iii) every band of $\hat{L}$ is $\hat{\gamma}$-closed. The order denseness of $L$ in $\hat{L}$ shows that every band of $\hat{L}$ containing $u$ must also contain $B_{u}$. Thus the smallest band containing $u$ in $\hat{L}$ is $\bar{B}_{u}$.

From the above corollary we might expect that the principal projection property is preserved under topological completion whenever $(L, \tau)$ is as in Corollary 7.3. It was shown by W. A. J. Luxemburg [7, Example 65. 6, p. 663] that this is not true and so Corollary 7.3 seems to be the best result we can get without additional assumptions.

Theorem 7.4. Let $(L, \gamma)$ be as in Corollary 7.3. Assume that $L$ bas sufficiently many projections. Then $\hat{L}$ bas sufficiently many projections.

Proof. Let $A$ be a nonzero band of $\hat{L}$. Then the order denseness of $L$ in $\hat{L}$ implies $A \cap L \neq\{\theta\}$ and that $A \cap L$ is a band of $L$. Pick a nonzero projection band $B$ of $L$ such that $B \subseteq A \cap L$ and use Lemma 1.3(iii) to see that $\bar{B} \subseteq A$. Now, apply Lemma 7.1 .

Theorem 7.5. Let $(L, \tau)$ be a Hausdorff locally solid Riesz space. Assume that $L$ is order dense in $\hat{L}$ and that $L$ has the projection property. Then $\hat{L}$ is Dedekind complete. 
Proof. Let $B$ be a band of $\hat{L}$. Then $B \cap L$ is a projection band of $L$. It follows from Lemma 7.1 that $\overline{B \cap L}$ is a projection band of $\hat{L}$. Note that Lemma 1.3(iii) implies $\overline{B \cap L} \subseteq B$. On the other hand if $\theta \leq \hat{u} \in B$ then $\theta \leq u_{\alpha} \uparrow \hat{u}$ holds in $\hat{L}$ for some net $\left\{u_{a}\right\}$ of $B \cap L$ and hence $\hat{u} \in \overline{B \cap L}$. Thus $B=\overline{B \cap L}$ and this shows that $\hat{L}$ has the projection property. The result now follows from $[9$, Theorem 42.6, p. 280] by observing that $\hat{L}$ is uniformly complete.

Remarks. (1) If $K$ and $L^{\delta}$ denote the universal and Dedekind completions of $L$ respectively and if $(L, \tau)$ is as in Theorem 7.5 then the following interrelationships hold: $L \subseteq L^{\delta} \subseteq \hat{L} \subseteq K$.

(2) Note that Theorem 7.5 is an extension of Theorem 2.6(ii).

8. Open problems. We conclude this paper by exhibiting some open questions:

(1) Are the $(A, i)$ and $(B, i)$ properties preserved under topological completion without assuming metrizability?

(2) Is Luxemburg's approximation theorem (Lemma 4.1) true without assuming metrizability? (Define $\theta \leq \hat{f} \in \hat{L}$ to be an upper element if $\theta \leq f_{a} \uparrow$ and $f_{a} \stackrel{\hat{q}}{\rightarrow} \hat{f}$ for some net $\left\{f_{a}\right\}$ of $L^{+}$.)

(3) Define the following generalized $(A, 0)$ property for locally solid Riesz spaces $(L, \tau): u_{a} \downarrow \theta$ and $\left\{u_{a}\right\}$ is a $\tau$-Cauchy net implies $u_{a} \stackrel{\tau}{\rightarrow} \theta$. Note that for metrizable spaces this is equivalent with $(A, 0)$ but not for nonmetrizable Riesz spaces (Example 1.2(v)). Note also that condition (ii) of Theorem 2.3 (Kawai's condition) implies this generalized $(A, 0)$ property. Question: Is Kawai's condition equivalent with the above property in general? Or in other words, is the generalized $(A, 0)$ property equivalent with the embedding of $L$ into $\hat{L}$ preserving arbitrary suprema and infima?

(4) Are the statements (iii) and (iv) of Theorem 6.1 equivalent for nonmetrizable Hausdorff locally solid Riesz spaces?

\section{REFERENCES}

1. C. D. Aliprantis, Some order and topological properties of locally solid linear topological Riesz spaces, Proc. Amer. Math. Soc. 40 (1973), 443-447.

2. M. Duhoux, Le complété d'un treillis localement solide, Rapport $\mathrm{n}^{\circ} 10$, avril 1971, Séminaires de Mathématique Pure, Institut de Mathématique Pure et Appliquée, Université Catholique de Louvain.

3. D. H. Fremlin, On the completion of locally solid vector lattices, Pacific J. Math. 43 (1972), 341-347.

4. J. Horváth, Topological vector spaces and distributions. Vol. I, Addison-Wesley, Reading, Mass., 1966. MR 34 \#4863. $\# 1902$.

5. I. Kawai, Locally convex lattices, J. Math. Soc. Japan 9 (1957), 281-314. MR 20

6. W. A. J. Luxemburg, Extensions of prime ideals and the existence of projections in Riesz spaces, Nederl. Akad. Wetensch. Proc. Ser. A 76 (1973), 263-279. 
7. W. A. J. Luxemburg, Notes on Banach function spaces, XVIa, XVIb, Nederl. Akad. Wetensch. Proc. Ser. A 68 = Indag. Math. 27 (1967), 646-667. MR 32 \#6202.

8. W. A. J. Luxemburg and A. C. Zaanen, Notes on Banach function spaces. II, IX, X, XIII, Nederl. Akad. Wetensch. Proc. Ser. A 66 = Indag. Math. 25 (1963), 148-153; ibid. A 67 = Indag. Math. 26 (1964), 360-376, 493-506, 530-543. MR 26 \#6723b; 30 \#3381a, b.

9. - Riesz spaces. I, North-Holland, Amsterdam, 1971.

10. A. L. Peressini, Ordered topological vector spaces, Harper and Row, New York, 1967. MR 37 \#3315.

11. H. Nakano, Semi-ordered linear spaces, Japan Society for the Promotion of Science, Tokyo, 1955. MR 17, 387.

12. H. H. Schaefer, Topological vector spaces, Macmillan, New York, 1966. MR 33 \#1689.

13. - On the completeness of topological vector lattices, Michigan Math. J. 7 (1960), 303-309. MR 23\#A2023.

DEPARTMENT OF MATHEMATICS, CALIFORNIA INSTITUTE OF TECHNOLOGY, PASADENA, CALIFORNIA 91109

Current address: Department of Mathematics, Occidental College, Los Angeles, California 90041 